(1)

CrossMark

\title{
Primary ciliary dyskinesia: the patients grow up
}

\author{
Claudia E. Kuehni ${ }^{1}$, Myrofora Goutaki ${ }^{1,2}$, Mary Carroll $^{3,4}$ and Jane S. Lucas ${ }^{3,4}$
}

Affiliations: ${ }^{1}$ Institute of Social and Preventive Medicine, University of Bern, Bern, Switzerland. ${ }^{2}$ Paediatric Respiratory Medicine, Children's University Hospital of Bern, University of Bern, Bern, Switzerland. ${ }^{3}$ Primary Ciliary Dyskinesia Centre, University Hospital Southampton NHS Foundation Trust, Southampton, UK. ${ }^{4}$ NIHR Southampton Respiratory Biomedical Research Unit, University of Southampton and University Hospital Southampton NHS Foundation Trust, Southampton, UK.

Correspondence: Claudia E. Kuehni, Institute of Social and Preventive Medicine, University of Bern, Finkenhubelweg 11, CH-3012, Bern, Switzerland. E-mail: claudia.kuehnidispm.unibe.ch

0 @ERSpublications

PCD is no longer a childhood disease and standards of care should be established from birth to late adulthood http://ow.ly/sORE300ZXI3

The study by SнAн et al. [1] in this issue of the European Respiratory Journal presents data from the largest study of adults with primary ciliary dyskinesia (PCD) described up to date. It asks important questions relating to the phenotype and prognosis of this rare disease. The article describes the practices of a single UK PCD centre, and since the patterns of referral and follow-up vary between clinics, is not representative of all adults with PCD. However, the data represent a wealth of clinical experiences and give us pointers for further studies.

The prevalence of PCD is unclear because symptoms are nonspecific and a gold standard diagnostic test is lacking [2-4]. Due to limited knowledge about this rare disease, patients commonly fail to be referred for diagnostic testing [5]. Furthermore, surveys among physicians [6] and patients [5] show that the procedures used to diagnose PCD vary widely. A survey of a previous PCD task force of the European Respiratory Society focusing on paediatric services identified 1192 patients across Europe, the majority aged $<20$ years [7]. Prevalence of diagnosed cases among 0-14-year-olds was one in 10000 to 20000 in some countries, but much lower in others. Median age at diagnosis was 5.3 years, with considerable between-country variation. Many young adults aged 20-30 years were still in paediatric care. Diagnostics and management varied between and within countries [6]. Children and adolescents (aged 0-19 years) account for $\sim 20 \%$ of the European population. As life expectancy in PCD is assumed to be near normal, we expect about four times as many adult PCD patients as children. This is in stark contrast to the paucity of publications on adults. In a systematic review, we searched for all papers reporting clinical characteristics of PCD patients, with a special focus on age-stratified data [8]. We found 17 studies describing paediatric populations, 32 describing populations of mixed age (including few, and mostly young, adults) and only three old studies focusing on adults [9-11].

Thus, patients with PCD seem to vanish from the research screen when they reach the age of 20 years. Are they not transitioned to adult pulmonology? Do they stop having scheduled visits? Do they die young?

\section{Novel data on PCD in adult patients}

The article by $\mathrm{SHAH}$ et al. [1] is timely. It comes from the Royal Brompton Hospital (RBH), London, UK, one of three designated national diagnostic centres for PCD in England [3, 12]. The RBH provides a diagnostic service for PCD for London and South East England, and offers specialist care for both

Received: June 012016 | Accepted: June 012016

Support statement: C.E. Kuehni and J.S. Lucas are participants in the European Union's Seventh Framework Programme under EG-GA number 35404 (BESTCILIA: Better Experimental Screening and Treatment for Primary Ciliary Dyskinesia). Funding information for this article has been deposited with the Open Funder Registry.

Conflict of interest: Disclosures can be found alongside this article at erj.ersjournals.com

Copyright OERS 2016 
paediatric and adult patients. The group has published widely on its paediatric patients [13-17] but this is their first report on adults. It presents data from 151 patients who had either been transitioned from the paediatric unit or had been diagnosed as adults. Median age at diagnosis was 23 years (range 0-72 years).

The manuscript provides important insights into the progression of severity of disease in adults with PCD. During the 7 years of follow-up, seven (4.6\%) persons died, five from respiratory causes. Of 93 patients who had undergone high-resolution computed tomography (HRCT) of the lungs, nearly all (95\%) had evidence of bronchiectasis. $45 \%$ of the study population were colonised by Pseudomonas aeruginosa. Lung function decline was estimated as $0.49 \%$ forced expiratory volume in $1 \mathrm{~s}$ (FEV1) per cent predicted per year. The authors studied factors associated with disease progression, and reported that older age at diagnosis, microtubular defects and HRCT scores predicted FEV1 decline.

The data presented in the article challenge common notions in PCD and reflect shortcomings in the available evidence. Several points are worthwhile highlighting. In keeping with a previous report from North America, FEV 1 decline was associated with microtubular defects; this might help us understand the pathogenesis of PCD and the way the defect is responsible for the functional abnormalities seen [18]. Pseudomonas colonisation was significantly more common in those with reduced lung function but did not predict subsequent worsening of lung function. As stated in the article, this might imply that Pseudomonas could be a marker of disease severity rather than a cause of disease progression. This fits with current thinking about the airway microbiome and is relevant to understanding pulmonary exacerbations not only in PCD but in other airway diseases, such as cystic fibrosis, bronchiectasis and chronic obstructive pulmonary disease $[19,20]$. The HRCT score is a sensitive measure of airway damage and decline. However, its repeated use is limited by the radiation conferred to the lungs. It is hoped that other techniques, such as magnetic resonance imaging, will provide similar information in the near future $[21,22]$.

\section{Transition from paediatric to adult care}

As with other chronic diseases that begin in childhood, we need a structured approach to prepare patients for transfer from family-centred paediatric clinics to adult clinics where patients must make informed decisions about their care. This process of transition should start some time before the transfer to adult services takes place. Cystic fibrosis shares many features with PCD including the need for intensive physiotherapy and antibiotics. Life expectancy in cystic fibrosis patients has increased over recent decades and it has changed from a predominantly paediatric disease to an adult one [23]. Models of transitional care for cystic fibrosis might inform PCD care; the need for collaborative working between paediatric and adult clinics is essential to enable longitudinal understanding of the patient "from cradle to grave". The

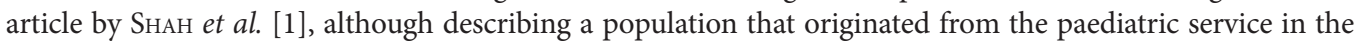
same hospital, does not draw on health status in childhood. The earliest information presented in the article dates from the time of transfer to adult services. Perhaps only patients, and not records, were transferred? Intensity of follow-up seems limited in this study. For instance, only 118 (78\%) out of 151 patients had had at least one lung function test performed and only $82(52 \%)$ patients had two or more lung function tests during the 7 years of follow-up. This seems little compared to cystic fibrosis, where 3-monthly lung function tests are recommended [24]. Perhaps the model of service delivery at the RBH, where the care of some patients is shared with nonspecialist pulmonology services, has led to a paucity of data.

\section{Does early diagnosis improve prognosis?}

Can good care after an early diagnosis prevent bronchiectasis and slow the decline of lung function? This question is difficult to answer using observational data because age at diagnosis is likely to be correlated with disease severity and more severely affected persons might be diagnosed earlier in life. Thus, these will seem to have a worse prognosis despite intensive therapy. MARThIN et al. [25], from the Danish National Reference Centre for PCD, looked at $>2900$ lung function measurements in 74 adult and paediatric PCD patients. During an observation period of 9.5 years, $34 \%$ of patients had a decline in lung function, $57 \%$ were stable and $10 \%$ improved. There was no evidence that courses of lung function were related to age at diagnosis or initial level of lung function [25]. The current study does not give clearer answers. Figure 2 of the article plots FEV1 at baseline against age at diagnosis. FEV1 at baseline was defined as the first lung function after diagnosis in patients diagnosed as adults or the lung function at the time of transition in patients diagnosed during childhood. This makes interpretation of the results difficult. The fact that FEV1 was lower in patients diagnosed later might be explained by the fact that those patients were older when their "baseline" lung function was taken. We cannot deduce how their lung function would be had they been diagnosed earlier. The question, whether good care can prevent the appearance of bronchiectasis and thereby improve patients' outcomes, can only be answered with truly prospective data from childhood onwards, ideally supported with randomised controlled studies that compare different therapies between groups of randomly allocated patients who do not differ in aspects other than the treatment in question. The first two randomised controlled trials in PCD, testing preventive treatments with azithromycin and hypertonic saline, are currently being conducted and results will be published soon [26]. 


\section{Is PCD a benign disease?}

The mortality data are hard to interpret, as they are not age standardised. However, nearly $5 \%$ of patients dying within 7 years seems high for an average age of 35 years. This challenges the notion that PCD does not affect life expectancy, and calls for larger studies with longer and complete follow-up for mortality. In our experience, the severity of disease in adults is highly variable but generally milder than in adults with cystic fibrosis. In Southampton, UK, we have patients who were diagnosed late in life but continue to live active and fulfilled lives in their 60s and 70s. Conversely, we care for significantly affected young adults because of impaired lung function and other aspects of this multisystem disease. Patients with congenital heart disease face increased risks and there have been case reports of PCD-related deaths even in children (personal communications and conference abstracts) [27]. Noone et al. [28] described severe disease progression in a significant minority of adults with PCD. Large representative data with long-term follow-up, such as the international PCD registry [29] or the international cohort study iPCD [30], will provide better mortality data in the future.

\section{Do “older patients" profit from new developments?}

Diagnosis of PCD is challenging and has changed over time. There is no single gold standard and the European Consensus guidelines (2009) recommend a combination of tests, including nasal nitric oxide (nNO), high-speed video microscopy (HSVMA) and transmission electron microscopy (TEM) [2]. The three diagnostic reference centres for PCD in England collaborate closely and diagnose PCD using a diagnostic algorithm based on nNO, HSVMA and TEM [3, 12]. Inconclusive and positive samples are reanalysed following culture of ciliated cells. Figure 1 of the article by SHAH et al. [1] suggests that this algorithm might only have been applied to newly diagnosed patients rather than reviewing diagnostic certainty periodically in the light of new developments in diagnostic testing. This is important given the lack of a gold standard diagnostic test and an evolving understanding of the diversity of the PCD phenotypes. The North American Disorders of Mucociliary Clearance Consortium reported half of the patients initially labelled as "PCD" were de-diagnosed after extensive testing [31]; conversely, we are aware of patients whose investigations had previously "excluded a diagnosis" where that decision has been overturned. In the study described by $\mathrm{SHAH}_{\text {et }}$ al. [1], nNO had been measured in only two-thirds of the patients (64\%), although it is a noninvasive and affordable test.

\section{What we can learn from this study}

The data presented from this adult PCD cohort add important evidence on the severity and long-term course of PCD in older patients. It is one piece of a large puzzle; data from other centres and more countries are highly desirable. We need prospective studies with complete and long-term follow-up from childhood throughout adult life and randomised controlled trials to give clearer answers to questions about mortality and progression of function, and how these can be improved. We must be faster in implementing new knowledge into ongoing clinical practice, such as applying new diagnostic standards to existing patients or applying transitional care. PCD is no longer a childhood disease and standards of care should be established from birth to late adulthood. There is a need for clinicians to collaborate to improve the experience of transition of patients and empower young adults to participate in decisions about their care. We propose that data "follow the patients" so that the adult pulmonologists are fully informed about patients' past history and contemporary information from shared-care centres. All this is vital to improve patient care, and understand and ameliorate prognosis.

\section{Acknowledgements}

All authors participate in the network of COST Action BEAT-PCD: Better Evidence to Advance Therapeutic options for PCD (BM 1407). C.E. Kuehni, M. Goutaki and J.S. Lucas are members of the European Respiratory Society Task Force for PCD Diagnostics (ERS TF-2014-04). The views expressed in this manuscript do not reflect official views of these collaborations.

\section{References}

1 Shah A, Shoemark A, MacNeill SJ, et al. A longitudinal study characterising a large adult primary ciliary dyskinesia population. Eur Respir J 2016; 48: 441-450.

2 Barbato A, Frischer T, Kuehni CE, et al. Primary ciliary dyskinesia: a consensus statement on diagnostic and treatment approaches in children. Eur Respir J 2009; 34: 1264-1276.

3 Lucas JS, Burgess A, Mitchison HM, et al. Diagnosis and management of primary ciliary dyskinesia. Arch Dis Child 2014; 99: 850-856.

4 Lucas JS, Walker WT, Kuehni CE, et al. Primary ciliary dyskinesia. In: Cordier J-F, ed. Orphan Lung Diseases (ERS Monograph). Sheffield, European Respiratory Society, 2011; pp. 201-217.

5 Behan L, Dunn G, Rubbo B, et al. Diagnosing primary ciliary dyskinesia; an international patient perspective. Eur Respir J 2016 [in press DOI: 10.1183/13393003.02018-2015].

6 Strippoli MP, Frischer T, Barbato A, et al. Management of primary ciliary dyskinesia in European children recommendations and clinical practice. Eur Respir J 2012; 39: 1482-1491. 
Kuehni CE, Frischer T, Strippoli MP, et al. Factors influencing age at diagnosis of primary ciliary dyskinesia in European children. Eur Respir J 2010; 36: 1248-1258.

8 Goutaki M, Meier A, Halbeisen F, et al. Clinical manifestations in primary ciliary dyskinesia: systematic review and metaanalysis. Eur Respir J 2016 [in press DOI: 10.1183/13393003.00736-2016].

9 Smit HJ, Schreurs AJ, Van den Bosch JM, et al. Is resection of bronchiectasis beneficial in patients with primary ciliary dyskinesia? Chest 1996; 109: 1541-1544.

10 Afzelius BA, Eliasson R. Male and female infertility problems in the immotile-cilia syndrome. Eur J Respir Dis Suppl 1983; 127: 144-147.

11 Camner P, Mossberg B, Afzelius BA. Measurements of tracheobronchial clearance in patients with immotile-cilia syndrome and its value in differential diagnosis. Eur J Respir Dis Suppl 1983; 127: 57-63.

12 Lucas JS, Chetcuti P, Copeland F, et al. Overcoming challenges in the management of primary ciliary dyskinesia: the UK model. Paediatr Respir Rev 2014; 15: 142-145.

13 Coren ME, Meeks M, Morrison I, et al. Primary ciliary dyskinesia: age at diagnosis and symptom history. Acta Paediatr 2002; 91: 667-669.

14 Karadag B, James AJ, Gültekin E, et al. Nasal and lower airway level of nitric oxide in children with primary ciliary dyskinesia. Eur Respir J 1999; 13: 1402-1405.

15 Maglione M, Bush A, Nielsen KG, et al. Multicenter analysis of body mass index, lung function, and sputum microbiology in primary ciliary dyskinesia. Pediatric Pulmonol 2014; 49: 1243-1250.

16 Irving SJ, Davies JC, Alton EW, et al. Lung clearance index in primary ciliary dyskinesia and bronchiectasis. Am J Respir Crit Care Med 2014; 189: 1147-1148.

17 Jain K, Padley SP, Goldstraw EJ, et al. Primary ciliary dyskinesia in the paediatric population: range and severity of radiological findings in a cohort of patients receiving tertiary care. Clin Radiol 2007; 62: 986-993.

18 Davis SD, Ferkol TW, Rosenfeld M, et al. Clinical features of childhood primary ciliary dyskinesia by genotype and ultrastructural phenotype. Am J Respir Crit Care Med 2015; 191: 316-324.

19 Pittman JE, Calloway EH, Kiser M, et al. Age of Pseudomonas aeruginosa acquisition and subsequent severity of cystic fibrosis lung disease. Pediatric Pulmonol 2011; 46: 497-504.

20 Smith EE, Buckley DG, Wu Z, et al. Genetic adaptation by Pseudomonas aeruginosa to the airways of cystic fibrosis patients. Proc Natl Acad Sci USA 2006; 103: 8487-8492.

21 Bauman G, Bieri O. Matrix pencil decomposition of time-resolved proton MRI for robust and improved assessment of pulmonary ventilation and perfusion. Magn Reson Med 2016 [in press DOI: 10.1002/mrm.26096].

22 Bauman G, Pusterla O, Bieri O. Ultra-fast steady-state free precession pulse sequence for Fourier decomposition pulmonary MRI. Magn Reson Med 2016; 75: 1647-1653.

23 Nazareth D, Walshaw M. Coming of age in cystic fibrosis - transition from paediatric to adult care. Clin Med (Lond) 2013; 13: 482-486.

24 Conway S, Balfour-Lynn IM, De Rijcke K, et al. European Cystic Fibrosis Society standards of care: framework for the cystic fibrosis centre. J Cyst Fibros 2014; 13: Suppl. 1, S3-22.

25 Marthin JK, Petersen N, Skovgaard LT, et al. Lung function in patients with primary ciliary dyskinesia: a cross-sectional and 3-decade longitudinal study. Am J Respir Crit Care Med 2010; 181: 1262-1268.

26 Paff T, Daniels J, Vonk-Noordegraaf A, et al. The effect of inhaled hypertonic saline on quality of life in primary ciliary dyskinesia patients: a double-blind randomized controlled cross-over trial. Am J Respir Crit Care Med 2016; 193: A1761.

27 Pittman J, Zariwala M, Weck K, et al. Respiratory failure as cause of death in an infant with primary ciliary dyskinesia. Am J Respir Crit Care Med 2011; 183: A1212.

28 Noone PG, Leigh MW, Sannuti A, et al. Primary ciliary dyskinesia: diagnostic and phenotypic features. Am J Respir Crit Care Med 2004; 169: 459-467.

29 Werner C, Lablans M, Ataian M, et al. An international registry for primary ciliary dyskinesia. Eur Respir J 2016; 47: 849-859.

30 Halbeisen F, Goutaki M, Maurer E, et al. Lung function in patients with primary ciliary dyskinesia (PCD): a multinational study. Eur Respir J 2015; 46: Suppl. 59: OA3480.

31 Leigh MW, Ferkol TW, Davis SD, et al. Clinical features and associated likelihood of primary ciliary dyskinesia in children and adolescents. Ann Am Thorac Soc 2016 [in press DOI: 10.1513/AnnalsATS.201511-748OC]. 